Fisioter Bras 2018;19(6):812-20

https://doi.org/10.33233/fb.v19i6.2502

\begin{abstract}
ARTIGO ORIGINAL
Mobilização precoce no paciente pediátrico criticamente enfermo: conhecimento e percepção da equipe multiprofissional de um hospital universitário

Early mobilization in critically ill pediatric patient: knowledge and perception of the multidisciplinary team of a university hospital
\end{abstract}

\author{
Beatriz da Silva Fagundes*, Cristiane Sousa Nascimento Baez Garcia**, Jairo Werner ${ }^{\star \star *}$ \\ *Universidade do Estado do Rio de Janeiro, **Docente e Suplente da Coordenação, Curso de \\ Graduação em Fisioterapia, Instituto Federal de Educação, Ciência e Tecnologia do Rio de \\ Janeiro - IFRJ, Campus Realengo, Rio de Janeiro/RJ, ${ }^{* * * U n i v e r s i d a d e ~ F e d e r a l ~ F l u m i n e n s e ~}$
}

Endereço para correspondência: Cristiane Sousa Nascimento Baez Garcia: cristiane.garcia@ifrj.edu.br; Beatriz da Silva Fagundes: biafag@yahoo.com.br, Jairo Werner: jairowerner@globo.com

\begin{abstract}
Resumo
Introdução: A falta de conhecimento sobre a mobilização precoce em pacientes pediátricos criticamente enfermos e a pouca percepção da sua importância têm sido descritos como contribuintes para a sua pouca difusão na área. Objetivo: Avaliar o conhecimento e a percepção da equipe multiprofissional de um hospital universitário acerca da mobilização precoce em pacientes pediátricos criticamente enfermos. Metodologia: Estudo observacional seccional analítico por meio de aplicação de questionário autopreenchido pelos profissionais da equipe multiprofissional que atuavam na Unidade de Terapia Intensiva Pediátrica de um hospital universitário. Resultados: 60 profissionais responderam ao questionário, dentre eles: cinco $(8,3 \%)$ fisioterapeutas, $19(31,7 \%)$ médicos, $12(20 \%)$ enfermeiros e $24(40 \%)$ técnicos em enfermagem. Entre os participantes, $35(58 \%)$ responderam que os estudos existentes na área pediátrica sugerem benefícios, enquanto $18(30 \%)$ relataram não ter opinião formada sobre o assunto. Com relação à percepção da importância da mobilização precoce, $14(23,3 \%)$ consideram-na crucial, $24(40 \%)$ muito importante, $18(30 \%)$ importante e quatro $(6,6 \%)$ pouco importante ou sem opinião sobre o assunto. Houve correlação positiva significativa entre 0 conhecimento e a percepção $(P=0,0075)$. Conclusão: $A$ maioria dos participantes possui conhecimento acerca do tema e reconhece a sua importância, existindo uma relação positiva entre essas variáveis.
\end{abstract}

Palavras-chave: reabilitação, unidades de terapia intensiva pediátrica, serviço hospitalar de fisioterapia.

\begin{abstract}
Introduction: The lack of knowledge about early mobilization in critically ill pediatric patients and the low perception of its importance have been described as contributing to its low diffusion in the area. Objective: To evaluate the knowledge and perception of the multiprofessional team of an university hospital about early mobilization in critically ill pediatric patients. Methodology: Analytical sectional observational study through the application of a self-administered questionnaire by professionals from the multiprofessional team working in the Pediatric Intensive Care Unit of an university hospital. Results: 60 professionals answered the questionnaire, among them: five (8.3\%) physiotherapists, 19 (31.7\%) physicians, $12(20 \%)$ nurses and $24(40 \%)$ nursing technicians. Of the participants, $35(58 \%)$ answered that existing studies in the pediatric area suggest benefits, while $18(30 \%)$ reported having no opinion about the subject matter. Regarding the perception of early mobilization importance, $14(23.3 \%)$ considered it to be crucial, $24(40 \%)$ very important, $18(30 \%)$ important and four (6.6\%) were of little importance or no opinion about the subject matter. There was a significant positive correlation between knowledge and perception $(P=0.0075)$. Conclusion: Most of the participants have knowledge about the topic and recognize its importance, and there is a positive relationship between these variables.
\end{abstract}

Key-words: rehabilitation, intensive care units, pediatrics, physical therapy department, hospital. 
Conceitualmente, o termo Mobilização Precoce (MP) se refere à aplicação de exercício físico nos primeiros dois a cinco dias da doença ou lesão grave, com intensidade suficiente para provocar alterações fisiológicas imediatas. Dentre elas, incluem-se melhora da ventilação, das perfusões periféricas e centrais, da circulação, do metabolismo muscular e do estado de alerta, além de atuar diretamente na prevenção da estase venosa e consequentemente da trombose venosa profunda [1].

No passado, os pacientes graves não eram considerados aptos para atividades físicas precoces porque eles eram avaliados como clinicamente instáveis ou dependentes de equipamentos vitais. Recentemente, evidências científicas contra essa argumentação têm sido publicadas [2,3]. Em 2007, o primeiro estudo de MP com pacientes submetidos à ventilação mecânica (VM) foi publicado e foram raros os eventos adversos relatados [3]. A viabilidade e segurança do procedimento também foram confirmadas por outros autores [4-6]. Em 2008, Morris et al. [4] mostraram por meio de estudo de coorte prospectivo que o início precoce da fisioterapia em pacientes criticamente enfermos está associado à redução do tempo de internação hospitalar e na Unidade de Terapia Intensiva (UTI). A partir daí, outros autores encontraram relação positiva entre a MP e a redução do tempo de internação na UTI/ hospitalar, inclusive em pacientes submetidos à VM [5,7-10].

A MP tem sido um tema amplamente estudado na abordagem intensiva de pacientes adultos. Algumas pesquisas demonstraram que esta é uma abordagem que pode diminuir 0 tempo de VM, de internação e de duração de delirium, além de melhorar o estado funcional do paciente e estar relacionada a menor mortalidade [11,12]. A importância da mobilização foi ainda reforçada por Gosselink et al. [13] nas recomendações da European Respiratory Society e European Society of Intensive Care Medicine como sendo uma intervenção de primeira linha para pacientes com disfunções do sistema cardiopulmonar.

Apesar de serem escassos os estudos de viabilidade e segurança da MP nos paciente pediátricos criticamente enfermos, os resultados sugerem que eles também estão aptos para tal prática [14-18]. Assim como na população adulta, na população pediátrica a MP parece trazer benefícios do ponto de vista funcional e de tempo de internação $[14,16,19,20]$. Entretanto, a prática da MP na pediatria ainda é pouco difundida e estimulada. Em estudo realizado no Canadá, verificou-se que apenas $3,4 \%$ dos Fisioterapeutas trabalhavam em uma UTIP que possuía protocolo de MP [21]. Em outro estudo retrospectivo realizado no mesmo país verificou-se que menos de $10 \%$ dos pacientes foram mobilizados precocemente [22]. A falta de conhecimento sobre o tema e a pouca percepção de sua importância tem sido descritos como contribuintes para esta realidade e a educação proposta como estratégia a ser utilizada para quebrar estas barreiras $[17,18]$.

Assim, o objetivo do presente estudo foi avaliar o conhecimento e a percepção da equipe multiprofissional de um hospital universitário (HU) acerca da MP em paciente pediátricos criticamente enfermos.

Tratou-se de um estudo observacional seccional analítico por meio da aplicação de questionário autopreenchido pelos profissionais da equipe multiprofissional que atua na UTIP de um HU localizado no município do Rio de Janeiro/RJ, Brasil. Tal unidade é composta por seis leitos, admitindo pacientes de um 0 a 18 anos incompletos com doenças clínicas ou cirúrgicas de todas as especialidades que já fazem acompanhamento ambulatorial no próprio hospital ou que chegam transferidas pela Central de Regulação do Estado. O período total do estudo compreendeu setembro de 2016 a maio de 2017.

Este estudo foi aprovado pelo Comitê de Ética em Pesquisa do próprio HU sob o número de CAAE 64110716.6.0000.5243. A participação no estudo foi precedida pela obtenção do termo de consentimento livre e esclarecido assinado pelo profissional.

Foram incluídos no estudo na qualidade de sujeito da pesquisa todos os profissionais dos diferentes seguimentos da área de Ciências da Saúde que atuavam na assistência ao paciente internado na UTIP do HU, incluindo fisioterapeutas, médicos, enfermeiros e técnicos de enfermagem. Além dos staffs e rotinas, os residentes de fisioterapia, medicina e enfermagem do segundo ano participaram da pesquisa. Residentes do primeiro ano foram excluídos por estarem atuando há apenas um mês na área no período da coleta de dados. 
Um questionário estruturado com perguntas fechadas foi elaborado para ser utilizado como instrumento nessa pesquisa. Seguindo as recomendações metodológicas, foi realizado o pré-teste do questionário preliminar em dezembro de 2016. Para isso, ele foi aplicado em amostra de conveniência composta de seis profissionais dos diferentes seguimentos da área de Ciências da Saúde que atuavam em outras unidades do HU ou em outros hospitais/ instituições. Com base no resultado do pré-teste, o questionário foi reformulado na sua apresentação e redação, com inclusão, mudança e exclusão de algumas perguntas. Como houve alterações significativas, foi necessário um novo pré-teste da segunda versão do questionário no mesmo mês. Assim sendo, a nova versão foi aplicada em outros quatro profissionais da área de Ciências da Saúde, não havendo nenhuma alteração pertinente a ser realizada. Essa versão foi definida, então, como a final do questionário semiestruturado a ser aplicada com os sujeitos da pesquisa.

As variáveis estudadas nesta pesquisa foram referentes a dois domínios presentes no questionário aplicado. O primeiro domínio avaliou o conhecimento do profissional sobre as evidências científicas existentes acerca dos benefícios da MP em pacientes pediátricos criticamente enfermos por meio de perguntas fechadas com gabarito pré-determinado. $O$ segundo domínio avaliou a percepção do profissional sobre a importância da MP nestes pacientes numa escala de 0 a 4, onde zero significa "nenhuma importância" e quatro "crucial", incluindo a opção de não ter opinião formada sobre o assunto. Dentre as variáveis, também foram incluídos o tempo de experiência em UTIP e a categoria profissional dos sujeitos da pesquisa.

\section{Coleta de dados}

Os dados foram coletados no período de 24 de março a 4 de abril de 2017. No período do estudo, atuava na UTIP do HU um total de 70 profissionais. Foram abordados pela pesquisadora, $63(90 \%)$ desses profissionais durante seu expediente de trabalho (turno diurno ou noturno) que, após explanação sobre o estudo, foram convidados a ler e, em caso de concordância na participação, assinarem o termo de consentimento livre e esclarecido. Não foi possível contatar sete profissionais durante o período de coleta dos dados.

Os participantes foram orientados a responderem os questionários individualmente, com sinceridade e clareza conforme cada enunciado, sem identificá-los sob nenhuma hipótese. O questionário foi respondido dentro do setor, no mesmo dia da explanação sobre o estudo e aceite para participação, conforme a disponibilidade de tempo do participante ao longo do plantão. A pesquisadora manteve-se distante durante o preenchimento dos questionários, em outro ambiente quando possível. Após o término do preenchimento, o participante dobrava o questionário e o depositava em uma urna de acrílico lacrada que permaneceu no setor durante todo o período de coleta de dados.

\section{Análise estatística}

Os dados foram tabulados como programa Excel® (versão 2007). Foi realizada a análise descritiva com medidas de tendência central e de distribuição de frequências. Para examinar a significância da associação entre as variáveis categóricas - conhecimento, percepção e "tempo de experiência", tabelas de contingência 2x2 foram construídas e os testes qui-quadrado e exato de Fisher aplicados. Foi considerado significativo o valor de $p<0,05$. $O$ software utilizado para desenvolver a análise foi o R.

Resultados

Dos 63 profissionais abordados, 60 responderam ao questionário, dentre eles, em ordem decrescente, 24 técnicos em enfermagem (40\%), 19 médicos $(31,7 \%), 12$ enfermeiros $(20 \%)$ e cinco fisioterapeutas $(8,3 \%)$. Além dos staffs e rotinas, dez residentes participaram da pesquisa (sete médicos - $70 \%$, dois enfermeiros - $20 \%$ e um fisioterapeuta - $10 \%$ ). A média de idade foi de 38 anos. Em relação ao tempo de experiência em UTIP, 47\% dos participantes possuíam menos de cinco anos e $53 \%$ cinco anos ou mais. 
Conhecimento sobre MP em pacientes pediátricos graves

Conforme observado na Tabela I, 58\% dos participantes apresentaram conhecimento de que as evidências científicas existentes apontam para os benefícios da MP em pacientes pediátricos criticamente enfermos. Vale comentar que 30\% dos profissionais disseram não ter opinião formada sobre 0 assunto (Tabela I).

Tabela I - Conhecimento da equipe multiprofissional sobre mobilização precoce em pacientes pediátricos criticamente enfermos.

Sobre a mobilização precoce em pacientes pediátricos graves, você acredita que: (marque somente uma opção)

a) Os estudos existentes sugerem benefícios. $35(58,3 \%)$

b) Não há evidência científica que suporte sua realização. $3(5 \%)$

c) Os estudos existentes sugerem que os riscos superam os $4(6,7 \%)$

d) Não tenho opinião formada sobre o assunto. $18(30 \%)$

Os valores estão expressos como frequência absoluta (frequência relativa).

Com relação ao conhecimento dos benefícios da MP em pediatria, existe diferença entre os diferentes segmentos profissionais da área de Ciências da Saúde. Todos os fisioterapeutas demonstraram conhecimento sobre o assunto, enquanto que entre os demais segmentos profissionais essa taxa caiu para pouco mais da metade (Tabela II).

Tabela II - Conhecimento dos diferentes seguimentos de profissionais da área de Ciências da Saúde sobre a mobilização precoce em pacientes pediátricos criticamente enfermos.

\begin{tabular}{lcccc}
\hline MP & Enfermeiro & Fisioterapeuta & Médico & $\begin{array}{c}\text { Técnico de } \\
\text { enfermagem }\end{array}$ \\
\hline Sem conhecimento & $5(41,7 \%)$ & $0(0 \%)$ & $9(47,4 \%)$ & $11(45,8 \%)$ \\
Com conhecimento & $7(58,3 \%)$ & $5(100 \%)$ & $10(52,6 \%)$ & $13(54,2 \%)$ \\
\hline Os valores estão expressos como frequência absoluta (frequência relativa). Sem conhecimento = somatório das \\
respostas B (não há evidência que suporte sua realização), C (os estudos sugerem riscos que superam os benefícios) \\
e D (não tenho opinião formada sobre o assunto).
\end{tabular}

Apesar das diferenças perceptíveis de distribuição de frequências entre as categorias profissionais com relação ao conhecimento da MP, aplicando-se o teste exato de Fisher não foi encontrada significância estatística $(P=0,28997)$.

Percepção da importância da MP em pacientes pediátricos graves

Com relação à percepção da importância da MP em pediatria, 18 profissionais (30\%) marcaram importante; 24 (40\%) muito importante; e 14 (23,3\%) crucial. Apenas 6\% dos participantes parecem não ter percepção da importância da MP no paciente pediátrico criticamente enfermo.

Observou-se ainda que a MP é vista com maior importância pelos fisioterapeutas (a maioria, $80 \%$, classificou como crucial) do que pelos demais segmentos de profissionais da equipe de saúde da UTIP do HU (a maioria dos médicos - 42,1\%, enfermeiros - 41,7\% e técnico de enfermagem - $41,7 \%$ classificou como muito importante). Além disso, comparativamente aos enfermeiros (16,7\% importante $+41,7 \%$ muito importante $+16,7 \%$ crucial $=75,1 \%)$, os técnicos de enfermagem $(41,7 \%$ importante $+41,7 \%$ muito importante + $12,5 \%$ crucial $=95,9 \%$ ) têm maior percepção da importância da prática da MP (Tabela III). 
Tabela III - Percepção da equipe multiprofissional e dos diferentes segmentos de profissionais da área de Ciências da Saúde sobre a importância da mobilização precoce em pacientes pediátricos criticamente enfermos.

Numa escala de 0 a 4 o quanto você acha importante a mobilização precoce do paciente pediátrico grave? (marque somente uma opção)

\begin{tabular}{llllll}
\hline Percepção & $\begin{array}{l}\text { Equipe } \\
(\mathbf{n}=\mathbf{6 0})\end{array}$ & $\begin{array}{l}\text { Fisioterapeuta } \\
(\mathbf{n}=\mathbf{5})\end{array}$ & $\begin{array}{l}\text { Médico } \\
(\mathbf{n}=\mathbf{1 9})\end{array}$ & $\begin{array}{l}\text { Enfermeiro } \\
(\mathbf{n = 1 2})\end{array}$ & $\begin{array}{l}\text { Técnico de } \\
\text { enfermagem } \\
(\mathbf{n}=\mathbf{2 4})\end{array}$ \\
\hline $\begin{array}{l}\text { Sem opinião } \\
\text { Pouco }\end{array}$ & $2(3,3 \%)$ & $0(0 \%)$ & $0(0 \%)$ & $2(16,7 \%)$ & $0(0 \%)$ \\
importante & $2(3,3 \%)$ & $0(0 \%)$ & $0(0 \%)$ & $1(8,2 \%)$ & $1(4,2 \%)$ \\
Importante & $18(30 \%)$ & $0(0 \%)$ & $6(31,6 \%)$ & $2(16,7 \%)$ & $10(41,7 \%)$ \\
$\begin{array}{l}\text { Muito } \\
\text { importante }\end{array}$ & $24(40 \%)$ & $1(20 \%)$ & $8(42,1 \%)$ & $5(41,7 \%)$ & $10(41,7 \%)$ \\
Crucial & $14(23,3 \%)$ & $4(80 \%)$ & $5(26,3 \%)$ & $2(16,7 \%)$ & $3(12,5 \%)$ \\
\hline
\end{tabular}

Os valores estão expressos como frequência absoluta (frequência relativa). Ninguém respondeu "Nenhuma importância".

Apesar das diferenças perceptíveis de distribuição de frequências entre as categorias profissionais com relação à percepção da importância da MP em pacientes pediátricos criticamente enfermos, aplicando-se o teste exato de Fisher não foi encontrada significância estatística $(P=0,25609)$.

Associação entre o conhecimento e a percepção da mobilização precoce em pacientes pediátricos graves

Analisando-se a relação entre o conhecimento e a percepção da importância da MP em crianças criticamente enfermas, por meio do teste exato de Fisher foi encontrada significância estatística $(P=0,0075)$, indicando assim a existência de associação entre as variáveis. Observando-se a Tabela IV, nota-se que quanto maior o conhecimento sobre a MP em pacientes pediátricos criticamente enfermos, maior a percepção sobre a sua importância.

Tabela IV - Associação entre conhecimento e percepção da importância da mobilização precoce em pacientes pediátricos criticamente enfermos.

\begin{tabular}{|c|c|c|c|c|c|c|}
\hline \multirow{3}{*}{$\begin{array}{l}\text { Percepção da } \\
\text { Importância }\end{array}$} & \multicolumn{6}{|c|}{ Conhecimento } \\
\hline & \multicolumn{2}{|c|}{ Frequência } & \multicolumn{2}{|c|}{$\%$ com coluna fixa } & \multicolumn{2}{|c|}{$\%$ com linha fixa } \\
\hline & Com & Sem & Com & Sem & Com & Sem \\
\hline Pouco/não importante & 1 & 3 & 3 & 12 & 25 & 75 \\
\hline Importante & 6 & 12 & 17 & 48 & 33 & 67 \\
\hline Muito importante & 16 & 8 & 46 & 32 & 67 & 33 \\
\hline Crucial & 12 & 2 & 34 & 8 & 86 & 14 \\
\hline
\end{tabular}

Tabela de contingência $2 \times 2$. Apresentação em sequência da esquerda para a direita de: frequência absoluta, frequência relativa com coluna fixa e frequência relativa com linha fixa. Considerado sem conhecimento sobre MP o somatório das respostas $\mathrm{B}$ (não há evidência que suporte sua realização), C (os estudos sugerem riscos que superam os benefícios) e D (não tenho opinião formada sobre o assunto).

Não houve associação do tempo de experiência em UTIP nem com o conhecimento ( $P$ $=0,32508$, teste qui-quadrado), nem com a percepção $(P=0,93668$, teste exato de Fisher $)$ sobre MP em crianças graves (Tabelas V e VI). 
Tabela V - Associação entre conhecimento sobre mobilização precoce (MP) em pacientes pediátricos criticamente enfermos e tempo de experiência em Unidade de Terapia Intensiva Pediátrica (UTIP).

\begin{tabular}{|c|c|c|c|c|c|c|}
\hline \multirow{3}{*}{$\begin{array}{l}\text { Conhecimento } \\
\text { sobre MP }\end{array}$} & \multicolumn{6}{|c|}{ Tempo de experiência em UTIP } \\
\hline & \multicolumn{2}{|c|}{ Frequência } & \multicolumn{2}{|c|}{$\%$ com coluna fixa } & \multicolumn{2}{|c|}{$\%$ com linha fixa } \\
\hline & $<5$ anos & $\geq 5$ anos & $<5$ anos & $\geq 5$ anos & $<5$ anos & $\geq 5$ anos \\
\hline $\begin{array}{l}\text { Com } \\
\text { conhecimento }\end{array}$ & 13 & 22 & 46 & 69 & 37 & 63 \\
\hline $\begin{array}{l}\text { Sem } \\
\text { conhecimento }\end{array}$ & 15 & 10 & 54 & 31 & 60 & 40 \\
\hline
\end{tabular}

Tabela de contingência 2x2. Apresentação em sequência da esquerda para a direita de: frequência absoluta, frequência relativa com coluna fixa e frequência relativa com linha fixa. Considerado sem conhecimento sobre MP o somatório das respostas B (não há evidência que suporte sua realização), C (os estudos sugerem riscos que superam os benefícios) e D (não tenho opinião formada sobre o assunto).

Tabela VI - Associação entre percepção sobre a importância da mobilização precoce (MP) em pacientes pediátricos criticamente enfermos e tempo de experiência em Unidade de Terapia Intensiva Pediátrica (UTIP).

\begin{tabular}{|c|c|c|c|c|c|c|}
\hline \multirow{3}{*}{$\begin{array}{l}\text { Percepção da } \\
\text { Importância da } \\
\text { MP }\end{array}$} & \multicolumn{6}{|c|}{ Tempo de experiência em UTIP } \\
\hline & \multicolumn{2}{|c|}{ Frequência } & \multicolumn{2}{|c|}{$\%$ com coluna fixa } & \multicolumn{2}{|c|}{$\%$ com linha fixa } \\
\hline & $<5$ anos & $\geq 5$ anos & $<5$ anos & $\geq 5$ anos & $<5$ anos & $\geq 5$ anos \\
\hline $\begin{array}{l}\text { Pouco } \\
\text { importante/ }\end{array}$ & 2 & 2 & 7 & 6 & 50 & 50 \\
\hline Importante & 9 & 9 & 32 & 28 & 50 & 50 \\
\hline $\begin{array}{l}\text { Muito } \\
\text { importante }\end{array}$ & 10 & 14 & 36 & 44 & 42 & 58 \\
\hline Crucial & 7 & 7 & 25 & 22 & 50 & 50 \\
\hline
\end{tabular}

Discussão

A pesquisa teve alta taxa de adesão (95\%) se comparada a demais semelhantes $[21,23]$. Tal fato pode ser explicado por: se tratar de um HU, onde há incentivo à produção científica; pelo vínculo existente entre a pesquisadora e demais membros da equipe ou pelo fato dos questionários não poderem ser levados para casa devendo ser respondidos durante o plantão do participante.

Infelizmente, a unidade possui equipe reduzida de fisioterapeutas, tendo um não participado da pesquisa e outro ser a própria pesquisadora. Assim, o número de participantes desse segmento profissional de Ciências da Saúde foi modesto, o que, além de prejudicar a interpretação dos resultados, dificultou a comparação com outros estudos.

Esse estudo é inovador em avaliar a temática em UTIP no Brasil e envolver os profissionais técnicos de enfermagem na abordagem da MP em pacientes graves. Os autores consideram a participação desse segmento da área de Ciências da Saúde fundamental para o sucesso da implantação de um programa de MP, visto que tais profissionais participam mais intensivamente do cuidado do paciente. Entretanto, a visão destes profissionais tem sido negligenciada nas pesquisas.

Do total de participantes, $42 \%$ desconheciam as evidências científicas relacionadas à MP em pacientes pediátricos criticamente enfermos. Interessantemente, em estudo semelhante realizado entre 2010 e 2012 no Canadá com apenas médicos e fisioterapeutas de 17 UTIP diferentes, essa taxa foi significativamente maior (87,5\%) [21]. De fato, o número de pesquisas sobre este tema, apesar de ainda escasso, cresceu nos últimos anos, o que provavelmente contribuiu para a difusão da prática [14-18]. Já em estudo com desenho similar realizado com profissionais que atuavam em UTI de pacientes adultos no mesmo país e período, essa taxa de falta de conhecimento acerca da MP foi bem menor, de $38 \%$, o que pode ser reflexo de maior número de publicações sobre MP em pacientes adultos criticamente enfermos [24].

A fraqueza muscular associada à UTI atinge aproximadamente $46 \%$ dos pacientes segundo revisão sistemática, podendo chegar até $100 \%$ em pacientes com quadro de sepse [25]. Entretanto, no estudo de Koo et al. [24] foi verificado que 68,8\% dos participantes, entre médicos e fisioterapeutas, subestimavam a incidência da fraqueza muscular adquirida em UTI. 
Acreditamos que esta taxa possa ser ainda maior na população pediátrica criticamente enferma, tendo em vista a dificuldade de ferramentas de avaliação adequadas para todas as faixas etárias desta população. No estudo publicado por Jolley et al. [23], aproximadamente $85 \%$ dos participantes, entre médicos, enfermeiros e fisioterapeutas, tinham conhecimento sobre os benefícios da MP na manutenção da força muscular e na redução do tempo de VM em pacientes adultos criticamente enfermos. Os autores desconhecem esses dados em pacientes pediátricos criticamente enfermos.

A importância da MP já foi atestada por inúmeros estudos. Além de estar relacionada à redução da prevalência do delirium, está associada ainda a outros benefícios como redução do tempo de VM, de internação hospitalar e aumento da funcionalidade [1]. No presente estudo, 93,3\% dos participantes reconhecem a importância da MP em pacientes pediátricos criticamente enfermos (tendo classificado como: importante; muito importante; ou crucial), sendo tal reconhecimento mais evidente entre os fisioterapeutas $(100 \%$, que a classificaram como muito importante ou crucial). No estudo similar realizado por Choong et al. [21] essa taxa foi de $76,1 \%$. Somando-se os que assinalaram que a MP é muito importante ou crucial, encontra-se uma taxa de 63,3\%, que é próxima a encontrada por Koo et al. [24] em seu estudo $(68,8 \%)$. Como a MP já é rotineiramente empregada nessa unidade local do estudo a percepção da importância de sua realização pode ser mais facilmente reconhecida pela equipe.

Entre os que identificaram a MP como uma prática crucial, a maioria $(80 \%)$ é composta por fisioterapeutas e a minoria $(12,5 \%)$ é de técnicos de enfermagem. Não parece ser surpresa a maior percepção da importância da MP entre os fisioterapeutas do que entre os demais segmentos da área de Ciências da Saúde, já que estes são os que comumente estudam tal prática em sua formação acadêmica.

No presente estudo, constatou-se que a percepção da importância está atrelada ao conhecimento, ou seja, quanto maior o conhecimento sobre as evidências científicas disponíveis sobre os benefícios da MP em pacientes criticamente enfermos, maior é a percepção da importância dessa abordagem. Não houve associação significativa entre o tempo de experiência na UTIP e estas variáveis (conhecimento e percepção da importância). Assim, parece claro que a difusão de conhecimento, seja em forma de publicações ou de programas educacionais, é fundamental para o devido reconhecimento da importância da MP pelos profissionais, incentivando assim a sua prática rotineira.

Os autores acreditam que unidades onde a implantação da rotina ou protocolo de MP seja penosa ou de difícil adesão possa ser reflexo da falta de conhecimento e, por conseguinte da percepção de importância por parte dos profissionais que nelas atuam.

$\mathrm{O}$ estudo tratou-se da análise específica de uma equipe multiprofissional que presta assistência na UTIP de um HU. Nessa equipe, muitos técnicos de enfermagem possuem também formação superior em enfermagem. Qualquer extrapolação dos resultados encontrados para demais unidades pode não ser adequada.

A mobilização precoce em pacientes pediátricos criticamente enfermos ainda não é uma prática tão difundida e a falta de conhecimento e de percepção sobre sua importância pode contribuir para essa defasagem. Quando se aplicou um questionário autopreenchido na equipe multiprofissional de uma UTIP, observou-se que mais da metade dos participantes possuem conhecimento sobre as evidências científicas acerca da mobilização precoce e poucos são os que não percebem a importância de sua realização. Além disso, verificou-se uma relação positiva entre as variáveis. Isso pode refletir na prática clínica nesse setor, onde a mobilização precoce é rotineiramente realizada e a aceita por toda equipe. Sugere-se, assim, que seja estimulada a educação continuada, assim como novos estudos na área, como forma de estimular a prática da mobilização precoce em pacientes pediátricos criticamente enfermos das UTIP de outros hospitais.

\section{Referências}

1. Cameron S, Ball I, Cepinskas G, Choong K, Doherty TJ, Ellis CG et al. Early mobilization in the critical care unit: A review of adult and pediatric literature. J Crit Care 2015;30(4):664-672. https://doi.org/10.1016/j.jcrc.2015.03.032 
2. Engel HJ, Needham DM, Morris PE, Gropper MA. ICU early mobilization: From recommendation to implementation at three medical centers. Crit Care Med 2013;41(Supl9):S69-S80. https://doi.org/10.1097/CCM.0b013e3182a240d5

3. Bailey P, Thomsen GE, Spuhler VJ et al. Early activity is feasible and safe in respiratory failure patients. Crit Care Med 2007;35:139-45. https://doi.org/10.1097/01.CCM.0000251130.69568.87

4. Morris PE, Goad A, Thompson C, Taylor K, Harry B, Passmore L et al: Early intensive care unit mobility therapy in the treatment of acute respiratory failure. Crit Care Med 2008;36:2238-43. https://doi.org/10.1097/CCM.0b013e318180b90e

5. Schweickert WD, Pohlman MC, Pohlman AS, Nigos C, Pawlik AJ, Esbrook CL, Spears $L$ et al: Early physical and occupational therapy in mechanically ventilated, critically ill patients: A randomised controlled trial. Lancet 2009;373:1874-82. https://doi.org/10.1016/S0140-6736(09)60658-9

6. Pohlman, MC, Schweickert WD, Pohlman AS, Nigos C, Pawlik AJ, Esbrook CL et al. Feasibility of physical and occupational therapy beginning from initiation of mechanical ventilation. Crit Care Med 2010;38(11):2089-94. https://doi.org/10.1097/CCM.0b013e3181f270c3

7. McWillians D, Weblin J, Atkins $\mathrm{G}$ et al. Enhancing rehabilitation of mechanically ventilated patients in the intensive care unit: A quality improvement project. J Crit Care 2015;30:13-8. https://doi.org/10.1016/i.jcrc.2014.09.018

8. Burtin C, Clerckx B, Robbeets C, Ferdinande P, Langer D, TroostersT et al. Early exercise in critically ill patients enhances short-term functional recovery. Crit Care Med 2009;37(9):2499-505. https://doi.org/10.1097/CCM.0b013e3181a38937

9. Feliciano VA, Albuquerque CG, Andrade FM, Dantas CM , Lopez A, Ramos FF et al. Influência da mobilização precoce no tempo de internamento na unidade de terapia intensiva. ASSOBRAFIR Ciência 2012;3(2):31-42.

10. Clark ED, Lowman JD, Griffin RL, Mathews HM, Reiff DA. Effectiveness of an early mobilization protocol in trauma and bruns intensive care unit: a retrospective cohort study. PhysTher 2013;93(2):186-96. https://doi.org/10.2522/pti.20110417

11. Schweickert WD, Kress JP. Implementing early mobilization interventions in mechanically ventilated patients in the ICU. Chest 2011;140(6):1612-7. https://doi.org/10.1378/chest.10-2829

12. Li Z, Peng X, Zhu B, Zhang Y, Xi X. Active mobilization for mechanically ventilated patients: a systematic review. Arch Phys Med Rehabil 2013;94:551-61. https://doi.org/10.1016/i.apmr.2012.10.023

13. Gosselink R, Bott J, Johnson M, Dean E, Nava S, Norrenberg M. Physiotherapy for adult patients with critical illness: recommendations of the European Respiratory Society and European Society of Intensive Care Medicine Task Force on Physiotherapy for Critically III Patients. Intensive Care Med 2008;34(7):1188-99. https://doi.org/10.1007/s00134-008-1026-7

14. Abdulsatar F, Walker RG, Timmons BW, Choong K. "Wii-Hab" in critically ill children: a pilot trial. J Pediatr Rehabil Med 2013;6:193-204. https://doi.org/10.3233/PRM-130260

15. Choong K, Chacon M, Walker R, Al-Harbi S, Clark H, Al-Mahr G et al. Early rehabilitation in critically ill children: a pilot study. Pediatr Crit Care Med 2014;15:8. https://doi.org/10.1097/01.pcc.0000448748.47635.b2

16. Hollander SA, Hollander AJ, Rizzuto S, Reinhartz O, Maeda K, Rosenthal DN. An inpatient rehabilitation program utilizing standardized care pathways after paracorporeal ventricular assist device placement in children. J Heart Lung Transplant 2014;33(6):587-92. https://doi.org/10.1016/j.healun.2013.12.009

17. Wieczorek B, Ascenzi J, Kim Y, Lenker H, Potter C, Shata NJ et al. PICU Up!: Impact of a quality improvement intervention to promote early mobilization in critically ill children. Pediatr Crit Care Med 2016;17(12):1-8. https://doi.org/10.1097/PCC.0000000000000983

18. Betters KA, Hebbar KB, Farthing D, Griego B, Easley T et al. Development and implementation of an early mobility program for mechanically ventilated pediatric patients. J Crit Care 2017;41:303-8. https://doi.org/10.1016/i.jcrc.2017.08.004

19. Jacobs BR, Salman BA, Cotton RT, Lyons K, Brilli RJ. Postoperative management of children after single-stage laryngotracheal reconstruction. Crit Care Med 2001;29(1):164-8. PMID: 11176178 
20. Melchers P, Maluck A, Suhr L, Scholten S, Lehmkuhl G. An Early onset rehabilitation program for children and adolescents after traumatic brain injury (TBI): methods and first results. Restor Neurol Neurosci 1999;14(2-3):153-60. PMID: 12671259

21. Choong K, Kool KK, Clark H, Chu R, Thabane L, Burns KE et al. Early mobilization in critically ill children: a survey of canadian practice. Crit Care Med 2013;41(7):1745-53. https://doi.org/10.1097/CCM.0b013e318287f592

22. Choong K, Foster G, Fraser DD, Hutchison JS, Joffe AR, Jouvet PA et al. Acute rehabilitation practices in critically ill children: a multicenter study. Pediatr Crit Care Med 2014;15(6):e270-279. https://doi.org/10.1097/PCC.0000000000000160

23. Jolley SE, Regans-Bagg J, Dickson RP, Hough CL. Medical intensive care unit clinician attitudes and perceived barriers towards early mobilization of critically ill patients: a cross-sectional survey study. BMC Anesthesiol 2014;14(84):1-9. https://doi.org/10.1186/1471-2253-14-84

24. Koo K, Choong K, Cook DJ, Herridge M, Newman A, Lo V. Early mobilization of critically ill adults: a survey of knowledge, perceptions and practices of Canadian physicians and physiotherapists. CMAJ open 2016;18;4(3):E448-E454. https://doi.org/10.9778/cmajo.20160021

25. Koo K, ChoongK, Faan E. Prioritizing rehabilitation strategies in the care of the critically ill. Critical Care Rounds 2011;8(4):1-7. 